\title{
Colposcopy Requirement of Papanicolaou Smear after Atypical Squamous Cells of Undetermined Significance (ASC-US) by Follow-up Protocol in an Urban Gynaecology Clinic, a Retrospective Study in Thailand
}

\author{
Thitichaya Perksanusak ${ }^{1}$, Panya Sananpanichkul ${ }^{1 *}$, Watcharin Chirdchim ${ }^{1}$, \\ Kornkarn Bhamarapravatana ${ }^{2}$, Komsun Suwannarurk ${ }^{3,4}$
}

\begin{abstract}
Background: ASC-US cases are managed according to the current American Society for Colposcopy and Cervical Pathology (ASCCP) guideline in which a human papillomavirus (HPV) test and repeat Pap smear are performed in the next 1 year. Colposcopy in cases of positive high risk HPV and persistent ASC-US or more in subsequent Pap smear is recommended. The HPV test is more expensive and still not currently a routine practice in Thailand. Objective: To identify the risk factors of persisted abnormal Pap smear and the colposcopic requirement rate in women with ASC-US. Materials and Methods: During 2008-2013, this study was conducted in Prapokklao Hospital, Chanthaburi, Thailand. Participants were women who attended gynaecology clinic for cervical cancer screening. Women who had cytological reports with ASC-US were recruited. During the study period, 503 cases were enrolled. Colposcopic requirement was defined as those who were detected with an ASC-US or more in subsequent Pap smears up to 1 year follow-up. Results: The colposcopic referral rate was 23.2 (85/365) percent at 12 months. Prevalence of cervical intraepithelial neoplasia (CIN) 2/3 was 3.3 (12/365) percent. Loss follow-up rate of subsequent Pap smear and colposcopic appointment were $27.4(138 / 503)$ and 48.2 (41/85) percent, respectively. There was no invasive cancer. High risk factors for persisted abnormal Pap smears in subsequent test were premenopausal status, HIV infected patients and non-oral contraceptive pills (COC) users. Conclusions: Referral rate for colposcopy in women with ASC-US reports was rather high. Loss to follow-up rate was the major limitation. Immediate colposcopy should be offered for women who had high risk for silent CIN.
\end{abstract}

Keywords: Papanicolaou smear - ASC-US - colposcopy

Asian Pac J Cancer Prev, 16 (12), 4977-4980

Introduction

Papanicolaou (Pap) smear is currently a screening method for early detection of cervical cancer. The most common cytologic abnormality was Atypical Squamous Cells of Undetermined Significance (ASC-US). The incidence of ASC-US was almost 5 percent with high spontaneous regression rate after repeat Pap smear at 4 months (Gerber et al., 2001). Persistent abnormal cytology was 16 percent (Gerber et al., 2001).

ASC-US was unequivocally reported and inconclusive management. According to the current American Society for Colposcopy and Cervical Pathology (ASCCP) guideline (Massad et al., 2012), HPV testing was preferred to repeat cytology testing at 12 months while the later still accepted. HPV testing arm was managed by colposcopic examination in case of ASC-US with high risk positive
HPV testing. Patients with conservative arm were operated by colposcopy if ASC-US persisted or more in subsequent 12 months Pap smear follow up. This was slightly different to previous ASCCP guideline that the triage of management ASC-US was immediate colposcopy, HPV testing and repeat Pap smear at 6 month interval (Thomas et al., 2006). The sensitivity and specificity of repeat Pap smear for ASC-US were only 15 and 93 percent while the sensitivity and specificity of directed colposcopic biopsy were 80 and 80 percent (Zarchi et al., 2011). Thus, directed colposcopic biopsy remained a better way for high grade lesion detection. Conservative arm can reduce colposcopic referral rate but delay the needed diagnosis and treatment.

The aim of study was to identify the risk factors of persisted abnormal Pap smear after ASC-US so that immediate colposcopy could be recommended in high risk cases.

${ }^{1}$ Department of Obstetrics and Gynaecology, Prapokklao Hospital, Chanthaburi, ${ }^{2}$ Department of Preclinical Science, ${ }^{3}$ Department of Obstetrics and Gynaecology, ${ }^{4}$ Chulabhorn International College of Medicine, Thammasat University, Pathum Thani, Thailand *For correspondence: panysanan@yahoo.com 


\section{Materials and Methods}

This was a retrospective study approved by the Ethic Committee of Prapokklao Hospital. All participants with ASC-US Pap smear from gynaecology clinic of Prapokklao Hospital, Chanthaburi, Thailand between January 2008 and December 2013 were recruited in this study.

Exclusion criteria were participants with previous abnormal Pap smear, post-hysterectomy or pregnant women.

Data were collected from medical records included age, occupation, residential location, menopausal status, family planning method, sexual behavior, type of cervical cytology screening and histopathology from colposcopic directed biopsy.

Routine protocol from management of ASC-US was followed according to current ASCCP guideline (Massad et al., 2012). The management was composed of HPV testing arm and conservative arm. HPV arm was managed by colposcopic examination in case of ASC-US with high risk HPV test positive. Conservative arm was operated by colposcopy in case of persisted ASC-US or more in subsequent Pap smear at 12 months follow-up periods. Conservative arm was used for general practice, while HPV testing still too expansive to routine used in this institute.

Women who had abnormal Pap smear in subsequent test were appointed to undergo colposcopic directed biopsy by gynaecologic oncologist expert. Cervical specimens were sent to be processed at Department of Pathology, Prapokklao Hospital Chanthaburi.

Statistical analysis was performed by using Statistical Package for the Social Sciences version 19.0 (SPSS, Chicago, IL, USA). Frequency, percentage, and the average mean were represented for demographic characteristics. Chi-square test and Fisher's exact test were used to compare categorical data. A t-test was used to compare continuous data. A p-value of less than 0.05 was defined as statistical significant.

\section{Results}

Five hundred and three women with ASC-US Pap smears were included in this study. Average of age was $38.4+11.4$ years (15-88 years). One sixth of cases in this study were nulliparous. Daily workers, housewives, merchants, civil servants, business employees, daily orchard labors and sex workers were 40.4, 23.7, 10.7, 8.7, 8.5 and 8 percent, respectively. Most of cases were Thai and of Buddhist religion.

One hundred and thirty eight cases $(27.4 \%)$ were missing from their subsequent Pap smear appointment. Three quarter (280/365) of cases who had undergone Pap smear as scheduled had negative for intraepithelial lesion or malignancy (NILM) in their cytological report. Remaining 85 cases had abnormal cytological report (ASC-US or more) and were further scheduled for colposcopic examination.

Table 1 showed baseline characteristics of all

Table 1. Baseline and Comparing Characteristics between Followed Up and Lossgroup for Subsequent Cervical Cytologic Test at 12 Months Follow-up Periods in Women with ASC-US at Prapokklao Hospital, Chanthaburi, Thailand

\begin{tabular}{|c|c|c|c|c|c|}
\hline \multicolumn{2}{|l|}{ Characteristic } & \multirow{2}{*}{$\frac{\mathrm{N}=503(\%)}{75(14.9)}$} & \multirow{2}{*}{$\begin{array}{c}\text { Loss follow up } \\
138(\%) \\
22(15.9)\end{array}$} & \multirow{2}{*}{$\begin{array}{l}\text { Follow up } \\
365(\%) \\
53(14.5)\end{array}$} & \multirow{2}{*}{$\frac{p \text {-value }}{0.676}$} \\
\hline Menopausal status & post-menopause & & & & \\
\hline & Pre-menopause & $428(85.1)$ & $116(84.1)$ & $312(85.5)$ & \\
\hline \multirow[t]{2}{*}{ Race } & Thai & 495 (98.4) & $134(97.1)$ & $361(98.9)$ & 0.224 \\
\hline & Others & $8(1.6)$ & $4 \quad(2.9)$ & $4(1.1)$ & \\
\hline \multirow[t]{2}{*}{ Religion } & Buddhist & $499(99.2)$ & $136(98.6)$ & $363(99.5)$ & 0.303 \\
\hline & Others & $4(0.8)$ & $2(1.4)$ & $2(0.5)$ & \\
\hline \multirow[t]{2}{*}{ Residential location } & Urban & $270(53.7)$ & $69(50)$ & $201(55.1)$ & 0.318 \\
\hline & Rural & $233(46.3)$ & $69(50)$ & $164(44.9)$ & \\
\hline \multirow[t]{6}{*}{ Occupation } & None & $119(23.7)$ & $38(27.5)$ & $81(22.2)$ & $0.140^{*}$ \\
\hline & Service & $203(40.4)$ & $49(35.5)$ & $154(42.2)$ & \\
\hline & Agriculture & $43(8.5)$ & $7(5.1)$ & $36 \quad(9.9)$ & \\
\hline & Government officer & $44 \quad(8.7)$ & $12(8.7)$ & $32(8.8)$ & \\
\hline & Merchant & $54(10.7)$ & $16(11.6)$ & $38(10.4)$ & \\
\hline & Sex worker & $40 \quad(8)$ & $16(11.6)$ & $24 \quad(6.6)$ & \\
\hline \multirow[t]{2}{*}{ Family Planning } & No & $148(29.4)$ & $39(28.3)$ & $109(29.9)$ & 0.744 \\
\hline & Yes & $355(70.6)$ & $99(71.7)$ & $256(70.1)$ & \\
\hline \multirow[t]{2}{*}{ COC contraception } & Yes & $66(13.1)$ & $14(10.1)$ & $52(14.2)$ & 0.241 \\
\hline & No & $437(86.9)$ & $124(89.9)$ & $313(85.3)$ & \\
\hline \multirow[t]{2}{*}{ Barrier contraception } & Yes & $135(26.8)$ & $35(25.4)$ & $100(27.4)$ & 0.735 \\
\hline & No & $368(73.2)$ & $103(74.6)$ & $265(72.6)$ & \\
\hline \multirow[t]{2}{*}{ HIV infection } & Yes & $163(32.4)$ & $36(26.1)$ & $127(34.8)$ & 0.07 \\
\hline & No & $340(67.6)$ & $102(73.9)$ & $238(65.2)$ & \\
\hline \multirow[t]{2}{*}{ Cytology technique } & Conventional Pap smear & $471(93.6)$ & $132(95.7)$ & $339(92.9)$ & 0.31 \\
\hline & Liquid based collection & $32(6.4)$ & $6 \quad(4.3)$ & $26(7.1)$ & \\
\hline \multirow[t]{2}{*}{ Cytology proposal } & Postpartum & $100(19.9)$ & $36(26.1)$ & $64(17.5)$ & $0.045^{* *}$ \\
\hline & Check-up & $403(80.1)$ & $102(73.9)$ & $301(82.5)$ & \\
\hline
\end{tabular}


Table 2. Characteristics of Participants in Colposcopic Study Needed according to Follow Up Protocol of ASC-US at 12 Months

\begin{tabular}{|c|c|c|c|}
\hline & \multicolumn{2}{|c|}{ Number of cases (\%) } & \multirow[t]{2}{*}{$\mathrm{p}$-value } \\
\hline & NILM & $(\geq$ AS-CUS) & \\
\hline Menopause & & & $<0.001 *$ \\
\hline Yes & $51(94.4)$ & $3 \quad(5.6)$ & \\
\hline No & $230(81.9)$ & $82(26.3)$ & \\
\hline Barrier contraception & & & $<0.001 *$ \\
\hline Yes & $62(62)$ & $38(38)$ & \\
\hline No & $219(82.3)$ & $47(17.7)$ & \\
\hline HIV & & & $0.002 *$ \\
\hline Yes & $85(66.9)$ & $42(33.1)$ & \\
\hline No & $196(82)$ & $43(18)$ & \\
\hline Cytology technique & & & 0.088 \\
\hline Conventional & $265(77.9)$ & $75(22.1)$ & \\
\hline Liquid based & $16(61.5)$ & $10(38.5)$ & \\
\hline Pap proposal & & & 0.193 \\
\hline Postpartum & $45(70.3)$ & 19 (29.7) & \\
\hline Checkup & $236(78.1)$ & $66(21.9)$ & \\
\hline Sexworker & & & 0.46 \\
\hline Yes & $17(70.8)$ & $7(29.2)$ & \\
\hline No & $264(77.2)$ & $78(22.8)$ & \\
\hline $\mathrm{COC}$ & & & $0.004 *$ \\
\hline Yes & 48 (92.3) & 4 (7.7) & \\
\hline No & $233(74.2)$ & $81(25.8)$ & \\
\hline
\end{tabular}

*NILM: negative for intraepithelial lesion or malignancy, Pap: Papanicolaou smear, ASC-US: atypical squamous cell of undetermined significance, HIV: human immunodeficiency virus, COC: combined oral contraceptive pills

Table 3. Correlation of Cervical Cytology and Histopathology

\begin{tabular}{lccccc}
\hline & No CIN & CIN1 & CIN 2/3 & Loss F/U & total \\
\hline ASC-US & 6 & 1 & 0 & 41 & 48 \\
LSIL & 14 & 11 & 8 & 0 & 33 \\
HSIL & 0 & 0 & 4 & 0 & 4 \\
Total & 20 & 12 & 12 & 41 & 85
\end{tabular}

*ASC-US: atypical squamous cell of undetermined significance, LSIL: low-grade squamous intraepithelial lesion, HSIL: high-grade squamous intraepithelial lesion, CIN: cervical intraepithelial neoplasia

participants. There were not significantly different of baseline characteristics between loss group and follow up group except postpartum status ( $\mathrm{p}$ value $<0.05$ )

The characteristics of participants in colposcopic study needed according to follow up protocol of ASC-US at 12 months were shown in Table 2. Participants with abnormal Pap smear ASC-US, LSIL and HSIL were classified as colposcopic candidates.

Prevalence of abnormal Pap smear at 12 months follow up for ASC-US, LSIL and HSIL patients were 56.5(48/85), $38.8(33 / 85)$ and $4.7(4 / 85)$, respectively. There was no invasive cancer found in this investigation. Nearly half of the cases (41/85) did not show up for their colposcopic appointment, all of which had ASC-US cytology reports. Correlation of cervical cytology and histopathology from colposcopy result were presented in Table 3 .

\section{Discussion}

From current ASCCP guideline (Massad et al., 2012), ASC-US patients received controversial management status. They could do HPV testing or conservative management (follow up with Pap smear). Meanwhile, immediate colposcopy was a histopathologic diagnostic test. Tatiyachonwiphut et al from Thailand reported the colposcopic diagnosis and cervical histopathology agreement was only 60\% (Tatiyachonwiphut et al., 2014). The colposcopy's cost more out of the pocket expense to the patients and required more sophisticated set up only available in some tertiary care medical facilities. Kececioglu's work from Turkey supported the HPV test rather than immediate colposcopy. HPV testing protocol gave higher CIN2/3 detection rate than immediate colposcopy arm. The total cost, cost per head and cost per CIN 2/3 detection were also higher than immediate colposcopy (Kececioglu et al., 2013). HPV testing utilized highly sophisticated set up with highest cost and took longer result turnaround time. HPV test has no longer to be cost effective compared to cytology test (Vanni et al., 2010). Therefore, conservative arm is the most popular choice at our situation due to availability and the cheapest cost for ASC-US management.

There were high percentage of lost Pap smear follow up and subsequent colposcopic appointment (26.4 and $48.2 \%$ respectively). Ek-anankul et al. studied first time Pap screening patients in rural area. Those that should receive free Pap smear but never took advantage of the program said they were not paying attention to the free screening because they had no gynecologic symptom (Ekanankul et al., 2009). They also did not want a stranger to approach their private female parts. From our study, our patients were subjected to their original Pap smear as a scheduled procedure, i.e., post natal Pap screening, or routine walk in. We believed that they did not care to come for a follow up because they had no physical symptom similar to Ek-anankul's finding. Loss of income on the appointment day was not the reason, as salary based and daily hired patients follow up rate showed no statistically significant outcome (data comparison not shown).

Most subsequent Pap smear results (Table 2) in postmenopausal population seem to be normal (NILM and $\geq$ ASC-US at 94.4 and 5.6 percent, respectively) while pre-menopausal group had more significant colposcopic need at one quarter of the population (81.9 and 26.3 percent, respectively). Tokmak et al reported statistically significant higher preinvasive lesions followed by ASCUS Pap smear in pre-menopausal women (Tokmak et al., 2014). Our finding supported Tokmak's result. As a result when ASC-US result was found in pre-menopausal population in a checkup a colposcopic protocol is highly recommended, while follow up is a preferred recommendation for post-menopausal subjects.

Women with barrier method contraception (condom) had significant lower spontaneous regress to NILM at 62\% than those with non-barrier contraception at $82.3 \%$ (Table 2). This finding supported the previous work of Chih et al, that stated that the condom usage showed no association with reduced risk for CIN (Chih et al., 2013).

HIV infected women had significantly higher repeat ASC-US finding in their follow up than that of the normal walk in population (33.1 and 18 percent, respectively) in similar manner to other studies as seen in Table 2 (Duerr 


\section{Thitichaya Perksanusak et al}

et al., 2005; Kodey et al., 2013). It was known that HIV infected patients was at high risks for abnormal cytology and needed stringent follow up with colposcopy after original ASC-US finding (Duerr et al., 2005; Kodey et al., 2013).

Table 2 showed lesser number of combined oral contraceptive pills (COC) users needed much less subsequent colposcopic procedures compared to nonuser group (7.7 and 25.8 percent, respectively). Chih et al claimed that the duration of COC use was inversely associated with CIN (Chih et al., 2013). Binesh et al reported no any specific association between COC consumption and abnormal Pap smear (Binesh et al., 2013). Our finding supported the above literatures.

Leung et al found out that sex workers had higher prevalence of abnormal Pap smear than normal walk in for Pap smear patients (Leung et al., 2013). In our finding sex workers as a group had no statistically significant outcome when regression or ASC-US at follow up was concerned in line with Leung's finding.

The prevalence of CIN2/3 from colposcopic examination was in Table 3 at $3.3(12 / 365)$ percent. The prevalence CIN2/3 rate from walk in checkup patients with colposcopic arm from Poomtavorn et al was 13.9 percent (Poomtavorn et al., 2011). Our prevalence was much lower compared to Poomtavorn study because we had high percentage of loss follow up.

Half of conservative management cases (280/503) had negative result in subsequent Pap smear and they had no need to undergo colposcopy. Conservative arm presented reduced colposcopic referral rate at around 55.6 percent. However, 16.9 (85/503) percent were affected by delayed colposcopy for diagnosis and treatment. Even though, CIN2/3 in this study was found only 3.28 percent, we suggested immediate colposcopic examination for patient with pre-menopausal status, HIV infection and non-combined oral contraceptive pills user when ASC-US status was found in walk in patients.

In conclusions, conservative management of ASC-US reduced half of colposcopic referral rate. One sixth of the cases were delayed diagnosis when treated by conservative protocol. Immediate colposcopy should be recommended in case of ASC-US with premenopausal status, HIV infected patient and non-combined oral contraceptive pills user.

\section{Acknowledgements}

Funding was supported by Research Institute of Prapokklao Hospital.

\section{References}

Binesh F, Akhavan Ali, Pirdehghan A, et al (2013). Does oral contraceptive pill increase the risk of abnormal Pap smear? Iran J Reprod Med, 11, 761-7.

Chih HJ, Lee AH, Colville L, et al (2014). Condom and oral contraceptive use and risk of cervical intraepithelial neoplasia in Australian women. J Gynecol onco, 25, 183-7.

Duerr A, Paramsothy P, Jamieson AJ, et al (2005). Effect of HIV infection on atypical squamous cells of undetermined significance. Clin Infect Dis, 42, 855-61.

Ek-anankul W, Promsen J, Sakdasri S (2009). Factors affecting cervical cancer screening by papanicolaou smear among women in Amphur Muang Nan, Nan Thailand. J Prapokklao Host Clin Med Educat Center, 26, 85-95.

Gerber S, Grandi PD, Petignat P, et al (2001). Colposcopic evaluation after a repeat atypical squamous cells of undetermined significance (ASC-US) smear. Int J Gynecol Obstet, 75, 251-5.

Kececioglu M, Seckin B, Baser E, et al (2013). Cost and effectiveness comparison of immediate colposcopy versus human papillomavirus DNA testing in management of atypical squamous cells of undetermined significance in Turkish women. Asian Pac J Cancer Prev, 14, 511-4.

Kodey PD, Narigapalli BP (2013). Conventional Pap smear screening in HIV seropositive women in South India. $J$ Obstet Gynaecol India, 63, 55-8.

Leung KM, Yeoh PS, Cheung HN, et al (2013). Prevalence of abnormal Papanicolaou smears in female sex workers in Hong Kong. Hong Kong Med J, 19, 203-6.

Massad LS, Einstein MH, Huh WK, et al (2012). 2012 Updated consensus guideline for management of abnormal cervical cancer screening tests and cancer precursors. 2012 ASCCP consensus guidelines conference. J Low Genit Tract Dis, 17, 1-27.

Poomtavorn Y, Suwannarurk K, Thaweekul Y, et al (2011). Risk factor for high-grade cervical intraepithelial neoplasia in patients with atypical squamous cells of undetermined significance (ASC-US) Papanicolaou smears. Asian Pac J Cancer Prev, 12, 235-8.

Tatiyachonwiphut M, Jaishuen A, Sangkarat S, et al (2014). Agreement between colposcopic diagnosis and cervical pathology: siriraj hospital experience. Asian Pac J Cancer Prev, 15, 423-6.

Thomas C, Wright Jr, Massad LS, et al (2006). 2006 Consensus guidelines for the management of women with abnormal cervical screening tests. J Low Genit Tract Dis, 11, 201-22.

Tokmak A, Guzel AI, Ozgu E, et al (2014). Clinical significance of atypical squamous cells of undetermined significance in detecting preinvasive cervical lesions in post-menopausal Turkish women. Asian Pac J Cancer Prev, 15, 6639-41.

Vanni T, Legood R, Franco EL, et al (2011). Economic evaluation of strategies for managing women Yazd. Asian Pac J Cancer Prev, 12, 3439-41.

Zarchi MK, Binesh F, Kazemi Z, et al (2011). Value of colposcopy in early diagnosis of cervical cancer in patients with abnormal pap smears at shahid sadoughi hospital, Yazd. Asian Pac J Cancer Prev, 12, 3439-41. 